\title{
Active Interference Constraint Learning with Uncertain Feedback for Cognitive Radio Networks
}

\author{
Anestis Tsakmalis, Symeon Chatzinotas and Björn Ottersten \\ SnT - securityandtrust.lu, University of Luxembourg \\ Email:\{anestis.tsakmalis, symeon.chatzinotas, bjorn.ottersten\}@uni.lu
}

\begin{abstract}
In this paper, an intelligent probing method for interference constraint learning is proposed to allow a centralized Cognitive Radio Network (CRN) to access the frequency band of a Primary User (PU) operating based on an Adaptive Coding and Modulation (ACM) protocol. The main idea is that the CRN probes the PU and subsequently applies a Modulation and Coding Classification (MCC) technique to acquire the Modulation and Coding scheme (MCS) of the PU. This feedback is an implicit channel state information (CSI) of the PU link, indicating how harmful the probing induced interference is. The intelligence of this sequential probing process lies on the selection of the power levels of the Secondary Users (SUs) which aims to minimize the number of probing attempts, a clearly Active Learning (AL) procedure, and consequently the overall PU QoS degradation. The enhancement introduced in this work is that we incorporate the probability of each feedback being correct into this intelligent probing mechanism by using a univariate Bayesian Nonparametric AL method, the Probabilistic Bisection Algorithm (PBA). An adaptation of the PBA is implemented for higher dimensions and its effectiveness as an uncertainty driven $A L$ method is demonstrated through numerical simulations.
\end{abstract}

Keywords-Cognitive Radio, Modulation and Coding Classification, Active Learning, Bayesian Nonparametric Inference, Probabilistic Bisection Algorithm

\section{INTRODUCTION}

The idea of Dynamic Spectrum Access (DSA) [1] is a key concept for flexible Radio Spectrum usage. Some DSA techniques suggest the use of frequency bands by unlicensed users (SUs) when the licensed ones (PUs) are absent or even their coexistence as long as the PU received interference is below a certain threshold. Cognitive Radio (CR) [2] is a candidate technology to reach this objective and enhance the operation of the SUs. In this paper, we focus on the coexistence scenario of PUs and SUs which is known within the CR context as the underlay scheme.

This vast topic has been thoroughly investigated from many aspects depending on the system model, the optimization variables, the objective functions and the constraints. An interesting approach of the underlay problem tackled by the research community is to consider a centralized CRN where a central decision maker, the Cognitive Base Station (CBS), elaborates an intelligent selection of the SU operational parameters, such as the transmit power levels, channels or time schedules, and communicates them through a control channel. In this context, network optimization problems have been formulated to achieve common or different SINR requirements for each
SU, maximum SU system throughput, maximum weighted throughput, maximum worst SU throughput or minimum transmit power, subject to PU QoS constraints, like $S I N R$, data rate or outage probability.

A major challenge though in all these underlay problems is the knowledge of the interference channel gains from the $\mathrm{CR}$ transmitters to the PU receivers and the PU receiver interference threshold which define the PU QoS constraints. This arises because PU systems usually depend on old communication technologies which were not foreseen to coexist and interact with other systems. Therefore, a CRN cannot rely on an access protocol that cooperates with the PUs in order to utilize their frequency bands. This lack of cooperation forbids passing information for inferring the interference channel gains and the PU interference threshold. Yet, the CRN must acquire some kind of knowledge about the CR-to-PU channel gains and the interference limit. A common approach to tackle this issue in the $\mathrm{CR}$ regime is the $\mathrm{CRN}$ to exploit a PU link state feedback, monitor how this changes because of the CRN operation and thus estimate these interference parameters. This idea, called proactive Spectrum Sensing (SS), was first proposed in [3] where the SU probes the PU and senses its effect from the PU power fluctuation, a rather informative piece of information. The proposed DSA application concerns only the SU system without adding any complexity in the infrastructure or a control channel between the PU system and the SU one in order to exchange information about the channel gains or the induced interference. Later work retained this probing scheme, but it focused on more rudimentary feedback such as the binary ACK/NACK packet of the reverse PU link [4-7] which requires the implementation of the complete PU receiver on the CRN side to decode the PU feedback message. The main problems of this approach are the hardware complexity of the complete PU receiver, security issues risen from the exploitation of the PU message and a minimum required SINR of the sensed PU signal to decode the PU message.

Another kind of implicit PU CSI, originally introduced in our previous work [8-10], is the MCC feedback which contains more information than the ACK/NACK packet and is easier to be extracted. For these case studies, the PU link is considered to be changing its MCS based on an Adaptive Coding and Modulation (ACM) protocol known to the coexisting system and the CRN is equipped with an MCC module to identify the modulation scheme and the code rate of the PU. The principal rationale of exploiting the MCC feedback in [8], [9] is to 
develop probing methods for interference channel gain and PU interference threshold learning with the minimum number of probing attempts. The ideal framework to achieve this goal is a Machine Learning field (ML) called AL. In [9], the devised AL method is performing multiple 1-D binary searches to estimate a hyperplane which depends on the interference parameters and defines the linear interference constraint of a CRN's operation. In [8], newly introduced AL techniques with reduced iteration complexity are used based on Cutting Plane Methods (CPMs), like the Analytic Center CPM and the Center of Gravity CPM, which are basically bisection methods in higher dimensions. On top of the greater learning speed, the latter AL techniques can perform well for learning the interference hyperplane while at the same time pursuing CRN capacity maximization, the optimization objective of the CRN's operation.

Fitting as this whole setting might be, there is still a practical consideration about the feedback that must be taken into account. In order to perfectly identify the MCC feedback, the MCC module on the CRN side must collect PU signal samples of adequate SNR. This case is usually not realistic and therefore the obtained information is not always reliable. This imperfect MCS identification depending on the SNR of the sensed PU signal samples is demonstrated in [10] and expressed quantitatively by the probability of correct classification $\left(P_{c c}\right)$. In contrast with likelihood based approaches, the MCC procedure developed in [10] also includes ML techniques which do not provide us a metric of how likely a group of PU signal samples corresponds to a specific MCS class. Therefore, modelling explicitly the error or the reliability of the MCC decision is not possible and the only way to derive a likelihood metric of the MCC feedback is in retrospect. This means that the correctness of this feedback can only be measured by taking into account performance empirical results of the MCC mechanism defined by $P_{c c}$ and depended on the SNR of the sensed PU signal.

In this paper, the probing design novelty is that the aforementioned uncertainty of the MCC feedback is taken into account within the AL framework described in [7], [8]. Nevertheless, regarding a discontinuous likelihood threshold like $P_{c c}$ as a reliability factor of the MCC feedback for an interference parameter learning method requires nonparametric inference methods. An additional reason for choosing nonparametric inference methods for our learning problem is that in AL the goal of minimizing the number of the training samples, here the probing power levels of the CRN, is achieved by forcing a selection policy for the training samples. This results in sample distributions not easily modelled and hence better expressed by nonparametric statistical models. An uncertainty driven AL technique for one dimension which is suitable for our problem setting is the PBA, a univariate Bayesian Nonparametric inference method [11]. In this work, an adaptation of the PBA is implemented for higher dimensions and used to intelligently probe a PU and learn the interference hyperplane with as less as possible probing attempts.

The remainder of this paper is structured as follows: Section II provides the system model. Section III analyzes the MCC feedback. Section IV introduces the probing algorithm for interference constraint learning based on a PBA adaptation.
Section V shows the results obtained by the application of the devised probing algorithm. Finally, Section VI gives the concluding remarks and future work in this topic.

\section{SySTEM MODEL}

Initially, the system model of the cognitive scenario must be described. Consider a PU link and $N$ SU links existing in the same frequency band as shown in Fig. 1. In addition, assume that the SUs belong to a centralized CRN where the CBS dictates through a control channel the SU operational parameters, such as their transmit power, and a multiple access method (e.g. FDMA) allows the SU links not to interfere with each other. Furthermore, the gains of the PU, interference and sensing channels are assumed to be static. In this study, we focus on channel power gains $g$ which in general are defined as $g=\|c\|^{2}$, where $c$ is the complex channel gain, and for brevity will be referred to as channel gains.

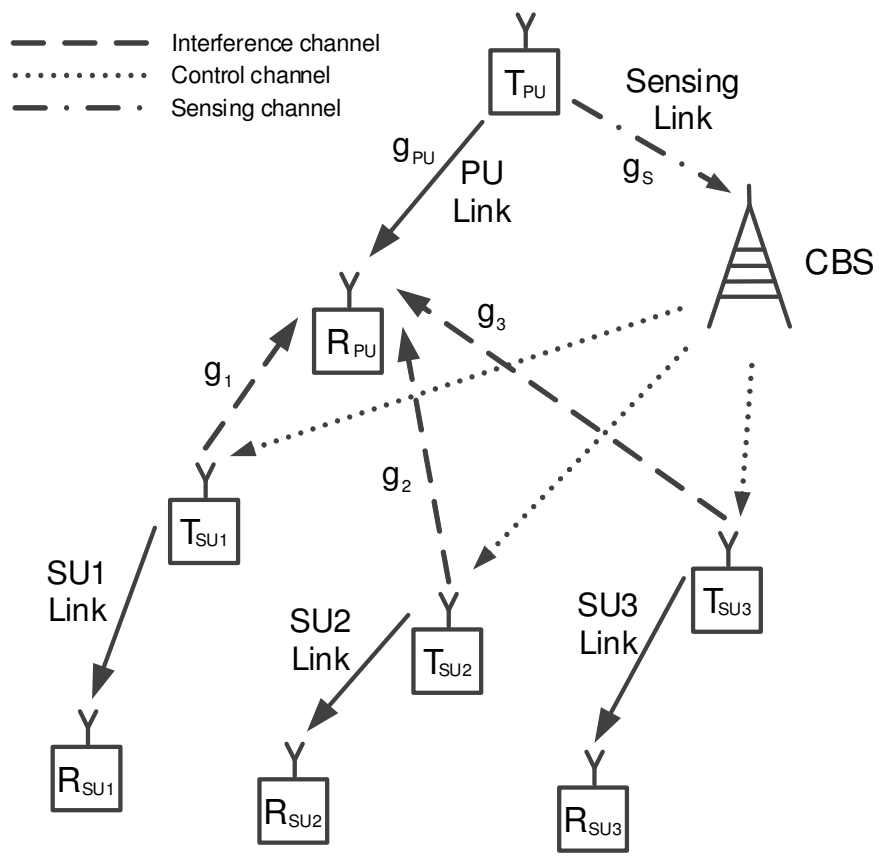

Fig. 1. The PU system and the CRN

The interference to the PU link is caused by the transmitter of each SU link to the receiver of the PU link. Taking into account that the SU links transmit solely in the PU frequency band, the aggregated interference on the PU side is defined as:

$$
I_{P U}=\mathbf{g}^{\top} \mathbf{p}
$$

where $\mathbf{g}$ is the interference channel gain vector $\left[g_{1}, \ldots, g_{N}\right]$ with $g_{i}$ being the $\mathrm{SU}_{\mathrm{i}}$-to-PU interference channel gain and $\mathbf{p}$ is the SU power vector $\left[p_{1}, \ldots, p_{N}\right]$ with $p_{i}$ being the $\mathrm{SU}_{\mathrm{i}}$ transmit power. Additionally, the SINR of the PU is defined as:

$$
S I N R_{P U}=10 \log \left(\frac{g_{P U} p_{P U}}{I_{P U}+N_{P U}}\right) \mathrm{dB}
$$


where $g_{P U}$ is the PU link channel gain, $p_{P U}$ is the PU transmit power and $N_{P U}$ is the PU receiver noise power.

Another important system aspect is that the CBS is equipped with a secondary omnidirectional antenna only for sensing the PU signal and an MCC module which enables it to identify the MCS of the PU. Here, it has to be pointed out that it is reasonable to assume that the $\mathrm{CRN}$ has some a priori knowledge of the standard of the legacy PU system whose frequency band attempts to enter and therefore the CRN can be aware of the PU ACM protocol. In this MCC process, introduced and described thoroughly in [10], an essential parameter is the SNR of the sensed PU signal:

$$
S N R_{S P U}=10 \log \left(\frac{g_{S} p_{P U}}{N_{C B S}}\right) \mathrm{dB}
$$

where $g_{S}$ is the sensing channel gain and $N_{C B S}$ is the noise power on the secondary CBS antenna side. A common condition is the $S N R_{S P U}$ level to be low and therefore causing imperfect MCC outputs.

\section{The Modulation And CODing Classification FEEDBACK AND ITS UNCERTAINTY}

In this section, we analyze the information given by the MCC feedback about the probing induced PU interference and define its reliability metric. Strong interference links may have a severe effect on the MCS chosen by the PU link and change it to more robust modulation constellations and coding rates depending on the level of the $S I N R_{P U}$. Let $\left\{M C S_{1}, . ., M C S_{J}\right\}$ denote the MCS set of the ACM protocol and $\left\{\gamma_{1}, . ., \gamma_{J}\right\}$ the corresponding minimum required $S I N R_{P U}$ values, which can also be expected to be known to the CRN as part of the PU ACM protocol and whenever violated, an MCS adaptation happens. Furthermore, consider these sets arranged such that $\gamma$ 's appear in an ascending order. Assuming that $N_{P U}$ and the received power remain the same at the PU receiver side, the $\left\{\gamma_{1}, . ., \gamma_{J}\right\}$ values correspond to particular maximum allowed $I_{P U}$ values, designated as $\left\{I_{t h_{1}}, . ., I_{t h_{J}}\right\}$. Hence, for every $M C S_{j}$ it can be inferred that $I_{P U}$ lies within the interval $\left(I_{t h_{j+1}}, I_{t h_{j}}\right]$, where $I_{t h_{j}}$ is the interference threshold over which the PU is obliged to change its transmission scheme to a lower order modulation constellation or a lower code rate and $I_{t h_{j+1}}$ is the interference lower limit below which the PU can change its transmission scheme to a higher order modulation constellation or a higher code rate. Still, the actual values of these thresholds are unknown to the CRN, since the CRN cannot be aware of the $N_{P U}$ and the received power at the PU receiver side.

Now, taking as reference the PU MCS when the CRN is silent and no probing occurs, $M C S_{\text {ref }}=M C S_{k}$, and the corresponding $\gamma_{\text {ref }}=\gamma_{k}$, where $k \in\{1, . ., J\}$, the following $\gamma$ ratios can be defined:

$$
c_{j}=\frac{\gamma_{j}}{\gamma_{\text {ref }}}
$$

where $j \neq k$ and $j \in\{1, \ldots, J\}$. Supposing a high $S N R_{P U}$ regime, $g_{P U} p_{P U} \gg N_{P U}$, the $I_{t h_{j}}$ ratios can also be determined as:

$$
\frac{I_{t h_{j}}}{I_{t h_{r e f}}}=\frac{\gamma_{r e f}}{\gamma_{j}}=\frac{1}{c_{j}}
$$

where $I_{t h_{\text {ref }}}$ is the interference threshold of $M C S_{r e f}$.

The knowledge of these ratios has a great significance for the final form of the MCC information. Let $M C S_{j}$ be the deteriorated MCS after the SU system probed the PU using an arbitrary SU power vector $\mathbf{p}$ within the feasible region $\Omega^{N}=$ $\left\{\mathbf{p} \mid 0 \leq p_{i} \leq p_{i}^{\max }, i=1, \ldots, N\right\}$. The information gained by the CBS as mentioned before is that:

$$
I_{t h_{j+1}}<\mathbf{g}^{\top} \mathbf{p} \leq I_{t h_{j}} .
$$

These inequalities can be rewritten using the $I_{t h}$ ratios as:

$$
\frac{I_{t h_{r e f}}}{c_{j+1}}<\mathbf{g}^{\top} \mathbf{p} \leq \frac{I_{t h_{r e f}}}{c_{j}} \Longleftrightarrow \frac{1}{c_{j+1}}<\tilde{\mathbf{g}}^{\top} \mathbf{p} \leq \frac{1}{c_{j}}
$$

where $\mathbf{g}$ is normalized with $I_{t h}=I_{t h_{r e f}}$ as $\tilde{\mathbf{g}}=\frac{\mathbf{g}}{I_{t h_{r e f}}}$. The former inequalities (7) can also be formulated in a further normalized version:

$$
\begin{aligned}
& \tilde{\mathbf{g}}^{\top} \tilde{\mathbf{p}}_{\mathbf{u}}>1 \\
& \tilde{\mathbf{g}}^{\top} \tilde{\mathbf{p}}_{\mathbf{l}} \leq 1
\end{aligned}
$$

where $\tilde{\mathbf{p}}_{\mathbf{l}}=c_{j+1} \mathbf{p}$ and $\tilde{\mathbf{p}}_{\mathbf{u}}=c_{j} \mathbf{p}$. In order to keep a single notation in (8) even for power vectors not degrading the PU MCS, the first inequality does not hold and $\tilde{\mathbf{p}}_{\mathbf{l}}$ is regarded equal to $\mathbf{p}$ in this special case.

The knowledge gained by the previous process and delineated in (8) is that first of all the MCC provides information about the unknown interference channel gain vector, $g$, and the interference threshold, $I_{t h_{r e f}}$, which define the PU QoS constraint:

$$
\mathbf{g}^{\top} \mathbf{p}=I_{t h_{r e f}}
$$

Specifically, the MCC output can be useful for learning the normalized to $I_{t h_{r e f}}$ version of (9):

$$
\tilde{\mathbf{g}}^{\top} \mathbf{p}=1
$$

which from the CRN operation point of view is equivalent to (9). Additionally, (8) represents a double piece of information as if two probing SU power vectors were applied. Furthermore, these power vectors $\tilde{\mathbf{p}}_{\mathbf{l}}$ and $\tilde{\mathbf{p}}_{\mathbf{u}}$ are closer than $\mathbf{p}$ to the unknown interference constraint (10) which means that any learning technique aiming to find (10) using probing power vectors and MCC feedback as training samples does not search uselessly the feasible region $\Omega^{N}$.

A final aspect of the MCC feedback playing important role in the learning approach of this paper is its uncertainty. As mentioned in the previous section, usually the $S N R_{S P U}$ level is not adequate to perfectly identify the PU MCS. Therefore, the depended on $S N R_{S P U}$ probability of correct MCS detection, which is expressed by the metric of $P_{c c}$ in [10], is most commonly below $100 \%$. The MCC procedure we developed in [10] though includes ML techniques which 
do not provide us a theoretically consistent metric of how likely a group of PU signal samples corresponds to a specific MCS class. Hence, we cannot explicitly model this reliability factor of the MCC feedback and a likelihood metric of the MCC output can only be estimated in retrospect. To accomplish that, performance empirical results of the MCC process showing the $P_{c c}$ dependence of each MCS to $S N R_{S P U}$ are required. However, this information is actually quite coarse and can only be used as a robust threshold likelihood function of the following form:

$$
\begin{aligned}
& P_{c c}=\operatorname{Pr}[M C S \mid \mathbf{g}]=\operatorname{Pr}\left[I_{t h_{j+1}}<\mathbf{g}^{\top} \mathbf{p} \leq I_{t h_{j}} \mid \mathbf{g}\right]= \\
& \operatorname{Pr}\left[\left(\tilde{\mathbf{g}}^{\top} \tilde{\mathbf{p}}_{\mathbf{u}}>1\right) \wedge\left(\tilde{\mathbf{g}}^{\top} \tilde{\mathbf{p}}_{\mathbf{l}} \leq 1\right) \mid \tilde{\mathbf{g}}\right]
\end{aligned}
$$

where $\wedge$ is the logical AND operator. In the next section, we investigate an AL method which considers this uncertainty information to infer (10) with the least probing attempts possible.

\section{ACTIVE LEARNING WITH UNCERTAIN FEEDBACK FOR PROBING}

From here on, the constraints (9) and (10) will be referred to as the interference hyperplane. Learning this hyperplane is the main topic of this section and to achieve it a strategy called proactive SS is employed where the CRN probes the PU and subsequently applies the MCC technique to monitor the PU MCS and collect the information described earlier. The target of this sequential probing and sensing process is to select SU power vectors which aim to minimize the number of probing attempts assuming the coarse uncertainty of the MCC feedback investigated earlier.

To this direction, an adaptation of a univariate Bayesian Nonparametric AL method, the PBA [11], for higher dimensions is implemented. In our previous work [9], a binary deterministic indicator based on MCS deterioration was exploited to perform binary searches on $N$ linear segments in order to find $N$ intersection points with the interference hyperplane as shown in Fig. 2 and hence the hyperplane itself. The enhancement proposed in this paper is first to manipulate better the MCC information as a multilevel feedback and secondly to include its uncertainty by using on the linear segments the PBA, a binary search like method which incorporates the uncertainty of each testing point.

First of all, to locate $N$ linear segments crossing the hyperplane, a number of end points needs to be known with some of them below the $N$-dimensional plane and the rest above it. Given that the interference hyperplane crosses the feasible region $\Omega^{N}$, there is always a known point below this $N$-dimensional plane, the $[0, \ldots, 0]$, and one above it, the $\left[p_{1}^{\max }, \ldots, p_{N}^{\max }\right]$. So, in the worst case scenario, $N-1$ more points are needed to define $N$ linear segments crossing the hyperplane. Now, if we consider that the initial sensing MCC feedback by the CRN when no probing occurs is $M C S_{r e f}$, a simple end point search is to examine random power vectors within $\Omega^{N}$ and check whether they cause or not PU MCS deterioration which indicates whether they are above or below the interference hyperplane respectively. After

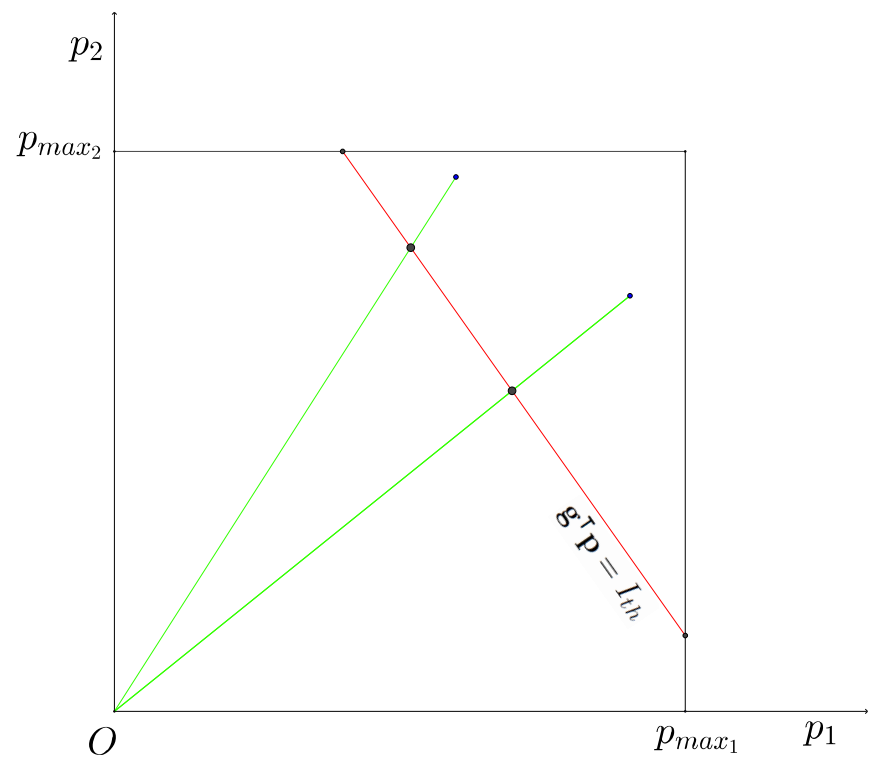

Fig. 2. A 2D graphical example where the intersection points of 2 randomly selected linear segments (green lines) and the interference hyperplane (red line) are shown

the end points are found and the line segments are defined, PBAs can be performed on each one of them so as to detect the $N$ intersection points and hence the interference hyperplane.

In brief, the PBA combines a univariate Bayesian Nonparametric inference method with the bisection algorithm and suggests that assuming a recursive Bayesian updating for estimating a 1-D parameter and an uncertain rudimentary feedback which indicates whether the true value of the 1-D parameter lies right or left of a testing point, the fastest way to learn its value is to always test next the median of the posterior derived from the sequential Bayesian updating. In order to use the PBA, a univariate parametric description of all the SU power vectors whose terminal points belong in a linear segment with arbitrary endpoints should also be given. Assuming the two endpoints as terminal points of the vectors $\mathbf{p}_{\mathbf{1}}$ and $\mathbf{p}_{\mathbf{2}}$, every point lying on the linear segment defined by these endpoints is expressed as terminal point of a vector $\mathbf{p}(\theta)$ using the parametric equation $\mathbf{p}(\theta)=\theta \mathbf{p}_{\mathbf{1}}+(1-\theta) \mathbf{p}_{\mathbf{2}}$, where $\theta \in[0,1]$. So, the problem comes down to estimating the vector $\mathbf{p}^{\text {in }}=\mathbf{p}\left(\theta^{*}\right)$ whose terminal point corresponds to the intersection point of the linear segment with the interference hyperplane. This can be tackled by performing the PBA on these linear segments and specifically on $N \theta$ regions to locate $N \theta^{*}$ s.

To describe in detail the recursive Bayesian updating, first we need to explain the role of (11) in this process. This uncertainty indicator in our case is not exactly binary like in the classical PBA. The information given by (11) in terms of where $\theta^{*}$ lies is:

$$
\begin{aligned}
& P_{c c}=\operatorname{Pr}\left[\left(\tilde{\mathbf{g}}^{\top} \tilde{\mathbf{p}}_{\mathbf{u}}>1\right) \wedge\left(\tilde{\mathbf{g}}^{\top} \tilde{\mathbf{p}}_{\mathbf{l}} \leq 1\right) \mid \tilde{\mathbf{g}}\right]= \\
& \operatorname{Pr}\left[\tilde{\mathbf{p}}_{\mathbf{l}} \preceq \mathbf{p}^{\mathbf{i n}} \preceq \tilde{\mathbf{p}}_{\mathbf{u}} \mid \mathbf{p}^{\mathbf{i n}}\right]=\operatorname{Pr}\left[\theta_{l} \leq \theta^{*}<\theta_{u} \mid \theta^{*}\right]
\end{aligned}
$$

where $\theta_{l}$ and $\theta_{u}$ are the $\theta$ values corresponding to $\tilde{\mathbf{p}}_{\mathbf{l}}$ and 
$\tilde{\mathbf{p}}_{\mathbf{u}}$ respectively. This expression is actually a robust threshold likelihood metric determined by the uncertainty of the MCC feedback, $P_{c c}$, which can also be written as:

$$
\begin{aligned}
& \operatorname{Pr}\left[\theta_{l} \leq \theta^{*}<\theta_{u} \mid \theta^{*}\right]= \\
& \operatorname{Pr}\left[M C S_{t} \mid \theta^{*}\right]=\left\{\begin{array}{cc}
P_{c c} & \text { if } \theta_{l}(t) \leq \theta^{*}<\theta_{u}(t) \\
1-P_{c c} & \text { otherwise }
\end{array} .\right.
\end{aligned}
$$

Now, let us describe thoroughly the recursive Bayesian updating process taking place in each linear segment. Following $(t-1)$ probing attempts, the CBS has collected $(t-1)$ MCC pieces of feedback, $M C S_{1:(t-1)}=\left\{M C S_{1}, . ., M C S_{(t-1)}\right\}$. After a new probing power vector $\mathbf{p}(t)$ which corresponds to $\theta_{l}(t)$ and $\theta_{u}(t)$, the $\theta^{*}$ posterior probability density function (pdf) according to Bayes rule is defined as:

$$
f_{t}\left(\theta^{*} \mid M C S_{1: t}\right)=\frac{\operatorname{Pr}\left[M C S_{t} \mid \theta^{*}\right] f_{t-1}\left(\theta^{*} \mid M C S_{1:(t-1)}\right)}{\operatorname{Pr}\left[M C S_{t} \mid M C S_{1:(t-1)}\right]}
$$

The denominator term is called the marginal likelihood and even though it appears difficult to be calculated, it is actually a normalization factor which guarantees that the posterior pdf integrates to 1 . Usually, it is computed as the integral of the numerator in (14) which in our case is a 1-D integration over the $\theta^{*}$ region. A general assumption when applying recursive Bayesian estimation and employed here as well is the prior pdf $f_{0}\left(\theta^{*}\right)$ for each linear segment PBA to be a uniform non informative pdf. This process is repeated until the uncertainty of $\theta^{*}$, expressed by the posterior pdf standard deviation $\sigma_{f_{t}}$, is below an accuracy threshold, our stopping criterion.

According to the PBA, in order to locate $\theta^{*}$ as fast as possible, the $\mathbf{p}(t+1)$ probing power vector should correspond to the $\theta(t+1)$ chosen as the median of $f_{t}\left(\theta^{*} \mid M C S_{1: t}\right)$ :

$$
\theta(t+1)=F_{t}^{-1}(1 / 2)
$$

where $F_{t}$ is the cumulative density function (cdf) of $f_{t}$. An important aspect of the PBA is its geometric convergence rate [11] proven to be $\mathcal{O}\left(\log _{c}\left(\frac{1}{\epsilon}\right)\right)$, where $1<c<2$.

Once, the PBA has been applied to all $N$ linear segments, the intersection points have been estimated and $\tilde{\mathrm{g}}$ can be found as the solution of an $N \times N$ system using the equality of the constraint (10):

$$
\tilde{\mathbf{g}}^{\top}=\left[\begin{array}{c}
\mathbf{p}_{1}^{\text {in }} \\
\mathbf{p}_{2}^{\text {in }} \\
\vdots \\
\mathbf{p}_{\mathbf{N}}^{\text {in }}
\end{array}\right]^{-1}\left[\begin{array}{c}
1 \\
1 \\
\vdots \\
1
\end{array}\right]
$$

where $\mathbf{p}_{\mathbf{i}}^{\mathbf{i n}}, i=1, \ldots, N$, are the probing power vectors as row vectors whose terminal points are the intersection points. An overview of the entire process described in this section is presented in Algo. 1.

\section{RESUlts}

Following, the performance of the probing process based on the described AL algorithm with uncertain feedback is presented. For testing the performance, a CRN with $N=3$

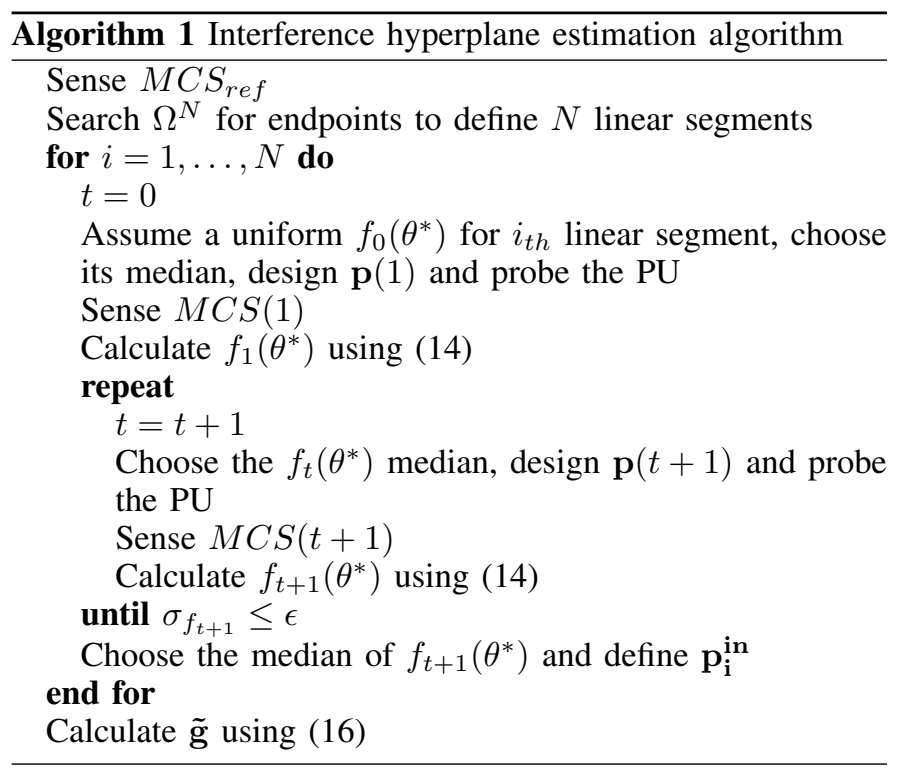

SUs is considered able to identify the correct PU MCS with $P_{c c}=0.6$. Initially, the $\theta^{*}$ posterior pdf updates for learning the first intersection point $\mathbf{p}_{1}^{\text {in }}$ are given in Fig. 3. In these diagrams, we observe that the uncertainty of the posterior pdf's is rapidly decreased as expected. Furthermore, erroneous MCC pieces of feedback cause multimodal posterior pdf's in early updating steps which in time center around the $\theta^{*}$ true value. Subsequently, the progress of each SU's power level $p_{i}$ is exhibited in Fig. 4 which represents the gradual convergence of the probing power vector coordinates to the coordinates of the intersection points $\mathbf{p}_{1}^{\mathrm{in}}$. We observe that a total number of 148 probing attempts is sufficient to learn the PU interference constraint even with a probability $P_{c c}=0.6$ of every MCC feedback being correct.

\section{CONCLUSIONS}

In this paper, an AL method using uncertain MCC feedback was developed for intelligent probing. The purpose of this process is enabling a centralized CRN to learn the interference constraint parameters of a PU in the context of an underlay cognitive scenario with the least number of probing attempts possible. Essentially, this is a recursive mechanism where the CRN intelligently probes the PU and subsequently applies an MCC technique to detect any PU MCS deterioration. To tackle imperfect MCS identification, the probability of each MCC feedback being correct was considered in this probing procedure by applying an adaptation of the PBA for higher dimensions. The proposed algorithm has proven geometric convergence rate which guarantees its fast performance even for large CRNs. Future work of this subject could be the development of probabilistic AL methods based on CPMs [7], [8] which consider uncertainty of the cutting planes.

\section{ACKNOWLEDGEMENT}

This work was supported by the National Research Fund, Luxembourg under the CORE projects "SeMIGod: SpEctrum 

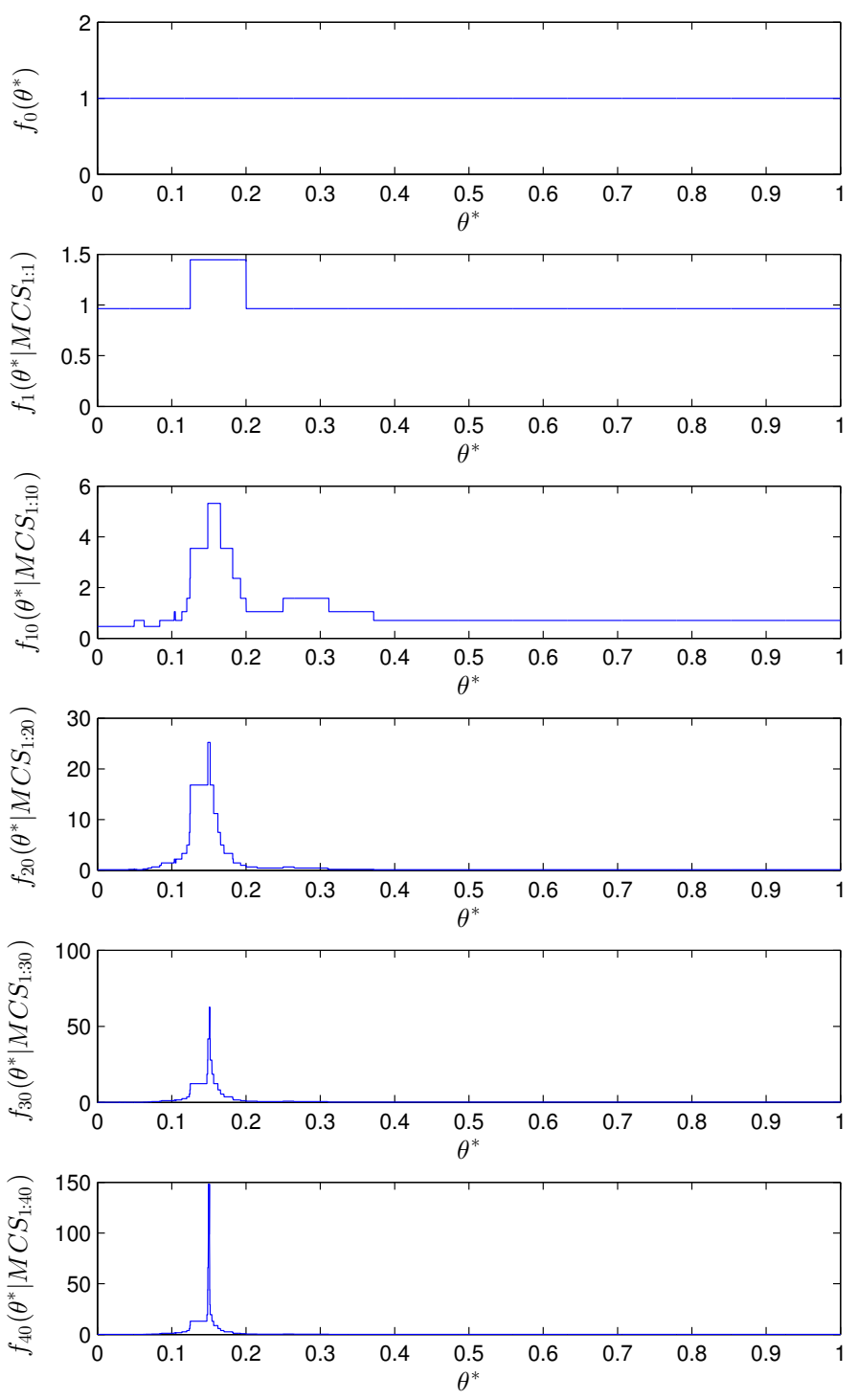

Fig. 3. $\theta^{*}$ posterior pdf updates with $P_{c c}=0.6$ after $1,10,20,30$ and 40 MCS pieces of feedback

Management and Interference mitiGation in cOgnitive raDio satellite networks" and "SATSENT: SATellite SEnsor NeTworks for spectrum monitoring".

\section{REFERENCES}

[1] Q. Zhao and B. Sadler, "A Survey of Dynamic Spectrum Access," IEEE Signal Process. Mag., vol. 24, no. 3, pp. 79-89, May 2007.

[2] J. Mitola, "Cognitive radio an integrated agent architecture for software defined radio," Ph.D. dissertation, KTH Royal Institute of Technology Stockholm, Stockholm, Sweden, 2000.

[3] G. Zhao, G. Y. Li, and C. Yang, "Proactive Detection of Spectrum Opportunities in Primary Systems with Power Control," IEEE Trans. Wireless Commun., vol. 8, no. 9, pp. 4815-4823, Sept. 2009.
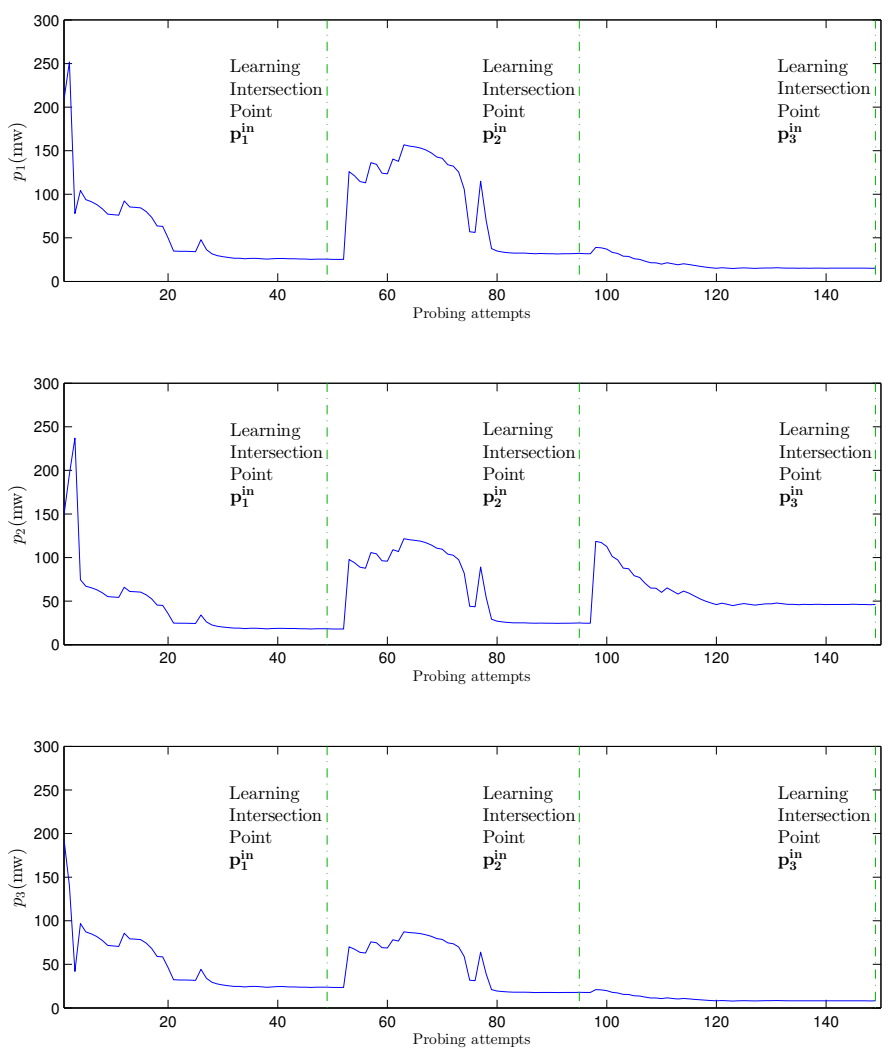

Fig. 4. Probing SU power levels for $N=3$ SUs

[4] S. Huang, X. Liu, and Z. Ding, "Decentralized Cognitive Radio Control Based on Inference from Primary Link Control Information," IEEE J. Sel. Areas Commun., vol. 29, no. 2, pp. 394-406, Feb. 2011.

[5] P. Zhou, Y. Chang, and J. Copeland, "Reinforcement Learning for Repeated Power Control Game in Cognitive Radio Networks," IEEE J. Sel. Areas Commun., vol. 30, no. 1, pp. 54-69, Jan. 2012.

[6] Y. Noam and A. J. Goldsmith, "The One-Bit Null Space Learning Algorithm and Its Convergence," IEEE Trans. Signal Process., vol. 61, no. 24, pp. 6135-6149, Dec. 2013.

[7] B. Gopalakrishnan and N. D. Sidiropoulos, "Cognitive Transmit Beamforming from Binary CSIT," IEEE Trans. Wireless Commun., vol. 14, no. 2, pp. 895-906, Feb. 2014.

[8] A. Tsakmalis, S. Chatzinotas, and B. Ottersten, "Centralized Power Control in Cognitive Radio Networks Using Modulation and Coding Classification Feedback," submitted to IEEE Trans. Cognitive Commun. and Networking, available on arXiv, 2015.

[9] A. Tsakmalis, S. Chatzinotas, and B. Ottersten, "Power Control in Cognitive Radio Networks Using Cooperative Modulation and Coding Classification," in Proc. 10th Int. Conf. on Cognitive Radio Oriented Wireless Netw. (CROWNCOM), Apr. 2015.

[10] A. Tsakmalis, S. Chatzinotas, and B. Ottersten, "Modulation and Coding Classification for Adaptive Power Control in 5G Cognitive Communications," in Proc. IEEE 14th Int. Workshop Signal Process. Adv. Wireless Commun. (SPAWC), Jun. 2014, pp. 234-238.

[11] R. Waeber, P. I. Frazier, and S. G. Henderson, "Bisection Search with Noisy Responses," SIAM J. Control Optim., vol. 51, no. 3, pp. 22612279, May 2013. 